\title{
THERE ARE NO INFINITE ORDER POLYNOMIALLY COMPLETE LATTICES AFTER ALL
}

\author{
MARTIN GOLDSTERN AND SAHARON SHELAH
}

\begin{abstract}
If $L$ is a lattice with the interpolation property whose cardinality is a strong limit cardinal of uncountable cofinality, then some finite power $L^{n}$ has an antichain of size $\kappa$. Hence there are no infinite opc lattices.

However, the existence of strongly amorphous sets implies (in $\mathrm{ZF}$ ) the existence of infinite opc lattices.
\end{abstract}

\section{INTRODUCTION}

We call a lattice $L n$-order polynomially complete (opc) if every monotone function $L^{n} \rightarrow L$ is induced by a lattice polynomial, and we say that $L$ is order polynomially complete if $L$ is $n$-order polynomially complete for every $n$.

This definition is from Schweigert's Ph.D. thesis [3]. The survey [2] gives several results and bibliographical references for results on order polynomially complete lattices.

While the finite opc lattices are now well understood, the main question on infinite opc lattices: are there any? has remained open until now.

We showed in [1] that the size of an infinite opc lattice (if one exists at all) must be a strongly inaccessible cardinal.

We now complement this result by showing (in ZFC) that the cardinality of an opc lattice cannot be a strongly inaccessible cardinal. Hence there are no infinite opc lattices.

Again the proof is not algebraic in nature, but based on a counting argument.

Unlike our previous proof, which employed the heavy machinery of partition calculus, this paper uses only very basic set theory (the notions of "cofinality" and "strong limit") and some baby model theory (the notion of "type").

We also point out that some version of $\mathrm{AC}$ (the axiom of choice) is necessary for our result, since under a strong negation of $\mathrm{AC}$ there are

Date: Oct 8, 1998.

1991 Mathematics Subject Classification. Primary 06A07; secondary 08A40, 06B99, 03E55.

Key words and phrases. polynomially complete, lattice, interpolation property, inaccessible cardinal, amorphous set, axiom of choice.

The second author is supported by the German-Israeli Foundation for Scientific Research \& Development Grant No. G-294.081.06/93. Publication number 688. 
pathological sets, which (while being infinite) are sufficiently similar to finite sets that it is still possible to build an opc lattice on them.

\section{No OPC LATTICES}

1.1. Definition. We say that a lattice $L$ has the unary interpolation property (1-IP), if every monotone function from $L$ into $L$ can be interpolated by a polynomial on any finite set. (Equivalently: Whenever $a, b, c, d \in L, b \not \leq a, c \leq d$, then there is a polynomial $p$ with $p(a)=c$, $p(b)=d$.)

1.2. Theorem. Assume that $L$ has the 1-IP. Let $\kappa$ be the cardinality of $L$ and assume that $\kappa$ is a strong limit cardinal of uncountable cofinality. Then for some natural number $n$ there is an antichain $A \subseteq L^{n}$ of cardinality $\kappa$.

Proof. Let $L$ be a lattice satisfying the assumptions of the theorem, and pick any two distinct comparable elements of $L$. We will call them 0 and 1 , where $0<1$.

We will define sequences $\left(L_{i}: i<\kappa\right),\left(a_{i}: i<\kappa\right),\left(b_{i}: i<\kappa\right)$, $\left(\bar{c}_{i}: i<\kappa\right),\left(n_{i}: i<\kappa\right),\left(\tau_{i}: i<\kappa\right)$ such that the following are satisfied for all $i, j<\kappa$ :

(1) $\{0,1\} \subseteq L_{i} \subseteq L,\left|L_{i}\right|<\kappa$

(2) If $i<j$, then $L_{i} \subseteq L_{j}$.

(3) $a_{i}$ and $b_{i}$ are in $L$ and realize the same (quantifier-free) type over $L_{i}$, i.e.: whenever $\sigma(\mathrm{x})$ and $\tau(\mathrm{x})$ are unary polynomials with coefficients in $L_{i}$, then $\sigma\left(a_{i}\right) \leq \tau\left(a_{i}\right)$ iff $\sigma\left(b_{i}\right) \leq \tau\left(b_{i}\right)$.

(4) $b_{i} \not \leq a_{i}$

(5) $n_{i}$ is a natural number

(6) $\tau_{i}$ is an $n_{i}+1$-ary term

(7) $\bar{c}_{i}=\left(c_{i}^{1}, \ldots, c_{i}^{n_{i}}\right) \in L^{n_{i}}$

(8) $\tau_{i}\left(a_{i}, \bar{c}_{i}\right)=0, \tau_{i}\left(b_{i}, \bar{c}_{i}\right)=1$.

(9) For all $i<j$ we have $a_{i}, b_{i}, c_{i}^{1}, \ldots, c_{i}^{n_{i}} \in L_{j}$.

The sequences are defined by induction on $i<\kappa$. In stage $i$ we let $L_{i}$ be the set of "everything" used so far: $L_{i}=\left\{0,1, a_{k}, b_{k}, c_{k}^{\ell}: k<\right.$ $\left.i, \ell \leq n_{k}\right\}$. Let $\lambda_{i}=\max \left(\left|L_{i}\right|, \aleph_{0}\right)$.

Since every type over $L_{i}$ can be represented as a set of pairs of polynomials with coefficients in $L_{i}$, there are at most $2^{\lambda_{i}}$ many possible types over $L_{i}$. By our assumption, $\lambda_{i}<\kappa$ implies $2^{\lambda_{i}}<\kappa$, so we can find two different elements $a_{i}, b_{i}$ which have the same type over $L_{i}$. Wlog $a_{i} \ngtr b_{i}$.

Now the function that maps the set $\left\{x: x \leq a_{i}\right\}$ to 0 and everything else (including $b_{i}$ ) to 1 is monotone, so it is realized by a polynomial $p_{i}(\mathrm{x})$. Let $n_{i}$ be the number of coefficients of $p_{i}$, so we can write $p_{i}(\mathrm{x})$ as $\tau_{i}\left(\mathrm{x}, c_{i}^{1}, \ldots c_{i}^{n_{i}}\right)$, where $\tau_{i}\left(\mathrm{x}, \mathrm{y}_{1}, \ldots, \mathrm{y}_{n_{i}}\right)$ is an $\left(n_{i}+1\right)$-ary term. This concludes the construction of our sequences. Note that if $\left(k_{i}\right.$ : $i<\kappa)$ is the increasing enumeration of a $\kappa$-size subset of $\kappa$, then $\left(L_{k_{i}}: i<\kappa\right),\left(a_{k_{i}}: i<\kappa\right)$, etc., also have all the properties listed above. 
Since $\kappa$ has uncountable cofinality, there must be some natural number $n$ such that $\left\{i: n_{i}=n\right\}$ has cardinality $\kappa$, so wlog (after thinning out our sequence, if necessary) we may assume that $n_{i}=n$ for all $n$. Similarly, since there are only countably many $n+1$-ary terms, we may assume all $\tau_{i}$ are equal to some fixed term $\tau$.

Now let $\bar{d}_{i}=\left(a_{i}, b_{i}, c_{i}^{1}, \ldots, c_{i}^{n}\right)$. We claim that $\left(\bar{d}_{i}: i<\kappa\right)$ is an antichain in $L^{n+2}$.

Indeed, pick any $i<j$ and assume that either $\bar{d}_{i} \leq \bar{d}_{j}$ or $\bar{d}_{i} \geq \bar{d}_{j}$. Since $\tau$ is monotone in each argument, we either have

$$
0=\tau\left(a_{i}, \bar{c}_{i}\right) \leq \tau\left(a_{j}, \bar{c}_{i}\right) \leq \tau\left(a_{j}, \bar{c}_{j}\right)=0
$$

or the converse inequality, so in any case $\tau\left(a_{j}, \bar{c}_{i}\right)=0$. Similarly we get $\tau\left(b_{j}, \bar{c}_{i}\right)=1$

However, since $a_{j}$ and $b_{j}$ have the same type over $\bar{c}_{i}, 0,1$, the equation $\tau\left(a_{j}, \bar{c}_{i}\right)=0$ implies $\tau\left(b_{j}, \bar{c}_{i}\right)=0$. This is a contradiction, so we conclude that $\bar{d}_{i}$ and $\bar{d}_{j}$ are incomparable.

\subsection{Conclusion. There is no infinite opc lattice}

Proof. Assume that $L$ is opc. A fortiori, $L$ has the 1-IP. Let $\kappa=|L|$. Since $L$ is opc, we know from [1] that $\kappa$ must be a strongly inaccessible cardinal, so in particular $\kappa$ is a strong limit cardinal of uncountable cofinality. By our theorem, there is an antichain $A \subseteq L$ of cardinality $\kappa$. But this easily implies that there are $2^{\kappa}>\kappa$ many monotone functions from $L^{n}$ to $L$, and at most $\kappa$ many of them can be polynomials.

\section{The ROLE OF AC}

2.1. Definition. An infinite set $A$ is called "strongly amorphous" if, for all natural numbers $n$, all $n$-ary relations on $A$ are first order definable (with parameters) in the language of equality. Equivalently, $A$ is amorphous if all sets $R \subseteq A^{n}$ are in the Boolean algebra generated by the sets $\left\{\left(x_{1}, \ldots, x_{n}\right): x_{i}=x_{j}\right\},\left\{\left(x_{1}, \ldots, x_{n}\right): x_{i}=a\right\}(a \in A)$.

While the axiom of choice (in fact, already a very weak version of $\mathrm{AC}$ ) clearly implies that there are no infinite strongly amorphous sets, it is well known that the theory " $\mathrm{ZF}+$ there is an infinite strongly amorphous set" is equiconsistent with ZFC. That is, ZF cannot refute the existence of infinite strongly amorphous sets. Hence (as we will see below), ZF cannot refute the existence of infinite opc lattices.

For the rest of this section we promise not to use the axiom of choice.

2.2. Theorem $(\mathrm{ZF})$. For every infinite set $L$ there is a bounded lattice $(L, \vee, \wedge, 0,1, \leq)$ such that:

For all natural numbers $n$, for all monotone functions $f$ :

$L^{n} \rightarrow L:$

If $f$ is definable in $(L, \vee, \wedge, \leq, 0,1)$, then $f$ is induced by a polynomial. 
2.3. Remark. (1) The "ZF" above means that this theorem is proved in the usual framework of mathematics (such as given by the Zermelo Fränkel axioms for the underlying set theory) but without invoking the axiom of choice.

(2) By "definable" we mean here: as a relation $f \subseteq L^{n+1}, f$ is definable by a first order formula (with parameters from $L$ ) in the language of lattice theory.

Since all our lattices will be bounded, it will be convenient to include the constants (or 0-ary operations) 0 and 1 into the "language of lattice theory". Thus, "definable with parameters $c_{1}, \ldots, c_{k}$ " will mean the same as "definable with parameters $c_{1}, \ldots, c_{k}, 0,1 "$.

2.4. Corollary (ZF). Assume that there is an infinite strongly amorphous set. Then there is an infinite order polynomially complete lattice.

2.5. Construction. Let $L$ be an infinite set, 0 and 1 two distinct elements of $L$. Define a lattice structure on $L$ by requiring $0 \leq a \leq 1$ for all $a \in L$.

2.6. Fact. Let $(L, \vee, \wedge, 0,1, \leq)$ be a lattice as in construction 2.5. Then every subset $R \subseteq L^{n}$ which is definable in $(L, \vee, \wedge, 0,1, \leq)$ with parameters $c_{1}, \ldots, c_{k} \in L$ is also definable (with parameters $c_{1}, \ldots, c_{k}, 0,1$ ) in the language of equality [i.e, in $(L,=)]$.

We will abbreviate a situation as in fact 2.6 by writing " $R$ is definable from $\left(c_{1}, \ldots, c_{k}\right)$." Functions $f: L^{n} \rightarrow L$ will be treated as relations $f \subseteq L^{n+1}$.

2.7. Notation. We indicate formal variables or indeterminates by a special typeface, e.g., $\mathrm{x}, \mathrm{t}_{1}$, etc.

We abbreviate tuples $\left(c_{1}, \ldots, c_{k}\right)$ and $\left(\mathrm{x}_{1}, \ldots, \mathrm{x}_{n}\right)$ as $\bar{c}^{k}$ and $\overline{\mathrm{x}}^{n}$, or sometimes $\bar{c}$ and $\overline{\mathrm{x}}$. We abbreviate $\left(\alpha, c_{1}, \ldots, c_{k}\right)$ by $(\alpha, \bar{c})$ or sometimes $\alpha, \bar{c}$.

$\operatorname{Mon}\left(L, L^{\prime}\right)$ is the set of all monotone maps from $L$ to $L^{\prime}$.

$\mathbb{T}$ is the set of all lattice-theoretic terms in the variables $\mathrm{x}_{1}, \mathrm{x}_{2}, \ldots$, $\mathbf{s}_{1}, \mathbf{s}_{2}, \ldots, \mathrm{t}_{1}, \mathrm{t}_{2}, \ldots$ (We include the constants 0 and 1 among "lattice-theoretic terms")

2.8. Definition. Assume that $L, L^{\prime}$ are isomorphic lattices, with isomorphism $\pi: L \rightarrow L^{\prime}$. We extend $\pi$ canonically to an isomorphism $\pi: L^{n} \rightarrow L^{n}$. For any $f: L^{n} \rightarrow L$ we write the conjugate function as $\pi f$ or $\pi(f)$ :

$$
\text { for all } \bar{a} \in L^{n}: \quad(\pi f)(\pi \bar{a})=\pi(f(\bar{a}))
$$

2.9. Fact. If $f: L^{n} \rightarrow L$ is definable from $\bar{c}=\left(c_{1}, \ldots, c_{k}\right), \pi: L \rightarrow L$ an automorphism which satisfies $\pi\left(c_{j}\right)=c_{j}$ for $j=1, \ldots, k$, then $\pi f=f$. 
2.10. Definition. Let $(L, \leq)$ be a partial order, $A \subseteq L$. The "monotone characteristic function of $A$ " is the function $\chi_{A}: L \rightarrow\{0,1\}$ defined by

$$
\chi_{A}(x)= \begin{cases}1 & \text { if } a \geq b \text { for some } b \in A \\ 0 & \text { otherwise }\end{cases}
$$

i.e., $\chi_{A}$ is the customary characteristic function of the upward closure of $A$.

From now on $L$ will be a lattice as in 2.5

2.11. Definition. Let $\left\{c_{1}, \ldots, c_{k}\right\} \subseteq L, \bar{d}=\left(d_{1}, \ldots, d_{m}\right) \in L^{m}$. We say that $\bar{d}$ is "independent over $\left\{c_{1}, \ldots, c_{k}\right\}$ " (or: over $\left(c_{1}, \ldots, c_{k}\right)$ ) iff all $d_{i}$ are distinct, and no $d_{i}$ is in $\left\{c_{1}, \ldots, c_{k}, 0,1\right\}$.

[This is a special case of the usual model-theoretic notion of independence.]

2.12. Definition. (1) Let $\chi\left(s, x, t_{1}, t_{2}, t_{3}\right)$ be the term

$$
\left[(x \wedge s) \vee t_{1}\right] \wedge\left[(x \wedge s) \vee t_{2}\right]
$$

(2) For $\alpha \in L \backslash\{0,1\}$ let $\chi_{\alpha}\left(\mathrm{x}, \mathrm{t}_{1}, \mathrm{t}_{2}, \mathrm{t}_{3}\right)=\chi\left(\alpha, \mathrm{x}, \mathrm{t}_{1}, \mathrm{t}_{2}, \mathrm{t}_{3}\right)$.

(3) If $A \subseteq\left\{c_{1} \ldots, c_{k}, 0,1\right\} \subseteq L$, then we define $\chi_{A}^{\bar{c}}\left(\overline{\mathrm{s}}^{k}, \mathrm{x}, \overline{\mathrm{t}}^{3}\right)$ (the "monotone characteristic function of $A$, given the parameters $\left.\vec{c}^{\prime \prime}\right)$ as follows:

(a) If $A=\emptyset$, then $\chi_{A}^{\bar{c}}\left(\overline{\mathrm{s}}, \mathrm{x}, \mathrm{t}_{1}, \mathrm{t}_{2}, \mathrm{t}_{3}\right)$ is the constant term 0 .

(b) If $A=\{1\}$, then $\chi_{A}^{\bar{c}}\left(\overline{\mathrm{s}}, \mathrm{x}, \mathrm{t}_{1}, \mathrm{t}_{2}, \mathrm{t}_{3}\right)=\mu\left(\mathrm{x} \wedge \mathrm{t}_{1}, \mathrm{x} \wedge \mathrm{t}_{2}, \mathrm{x} \wedge \mathrm{t}_{3}\right)$, where $\mu$ is the following "majority term":

$$
\mu(\mathrm{x}, \mathrm{y}, \mathrm{z})=(\mathrm{y} \vee \mathrm{z}) \wedge(\mathrm{z} \vee \mathrm{x}) \wedge(\mathrm{x} \vee \mathrm{y})
$$

(c) If $0 \in A$, then $\chi_{A}^{\bar{c}}\left(\overline{\mathrm{s}}, \mathrm{x}, \mathrm{t}_{1}, \mathrm{t}_{2}, \mathrm{t}_{3}\right)$ is the constant term 1 .

(d) Otherwise we let $I=I_{A}^{\bar{c}}=\left\{i: c_{i} \in A \backslash\{0,1\}\right\}$ and we let

$$
\chi_{A}^{\bar{c}}(\mathrm{x}, \overline{\mathrm{t}})=\bigvee_{i \in I} \chi_{c_{i}}(\mathrm{x}, \overline{\mathrm{t}})
$$

(4) If $A$ is cofinite, $L \backslash A \subseteq\left\{c_{1}, \ldots, c_{k}\right\}$, then we define $\chi_{A}^{\bar{c}}(\overline{\mathrm{s}}, \mathrm{x}, \overline{\mathrm{t}})$ similarly (dually), such that fact 2.13 below holds. We leave the details to the reader.

2.13. Fact. If $A \subseteq\left\{c_{1}, \ldots, c_{k}, 0,1\right\}$ or $L \backslash A \subseteq\left\{c_{1}, \ldots, c_{k}, 0,1\right\}$, and $\bar{d}=\left(d_{1}, d_{2}, d_{3}\right)$ is independent over $c_{1}, \ldots, c_{k}$, then the function

$$
a \mapsto \chi_{A}^{\bar{c}}\left(\bar{c}, a, d_{1}, d_{2}, d_{3}\right)
$$

is the monotone characteristic function of $A$.

Proof. Easy computation.

2.14. Lemma. Let $L$ be as in construction 2.5. Then there is a function

$$
p:\left(\bigcup_{n=0}^{\infty} \operatorname{Mon}\left(L^{n}, L\right)\right) \times\left(\bigcup_{k=1}^{\infty} L^{k}\right) \rightarrow \mathbb{T}
$$


that assigns to each pair $(f, \bar{c})$ a polynomial $p^{f ; \bar{c}} \in \mathbb{T}$ such that the following hold:

(1) If $\bar{c}=\left(c_{1}, \ldots, c_{k}\right)$ and $f: L^{n} \rightarrow L$, then $p^{f ; \bar{c}}$ is a term using (at most) the variables $\mathrm{s}_{1}, \ldots, \mathrm{s}_{k}, \mathrm{x}_{1}, \ldots, \mathrm{x}_{n}, \mathrm{t}_{1}, \ldots, \mathrm{t}_{3^{n}}$. We will write $p^{f ; \bar{c}}$ as $p^{f ; \bar{c}}(\overline{\mathbf{s}} ; \overline{\mathbf{x}} ; \overline{\mathbf{t}})$.

(2) If $\bar{c}$ and $f$ are as above, and $f$ is definable from $c_{1}, \ldots, c_{k}$, then we have:

Whenever $\bar{d}=\left(d_{1}, \ldots d_{3^{n}}\right)$ is independent over $\left\{c_{1}, \ldots, c_{k}\right\}$, then for all $\bar{a} \in L^{n}: f(\bar{a})=p^{f ; \bar{c}}(\bar{c} ; \bar{a} ; \bar{d})$, that is, the function $f$ is induced by the lattice polynomial $p^{f ; \bar{c}}(\bar{c} ; \overline{\mathbf{x}} ; \bar{d})$.

(3) Moreover, the term $p^{f ; \bar{c}}$ depends only on the "isomorphism type" of $(f, \bar{c})$. That is, whenever $\pi: L \rightarrow L^{\prime}$ is an isomorphism, then $p^{f ; \bar{c}}(\overline{\mathbf{s}}, \overline{\mathbf{x}}, \overline{\mathrm{t}})=p^{\pi f, \pi(\bar{c})}(\overline{\mathbf{s}}, \overline{\mathbf{x}}, \overline{\mathrm{t}})$.

2.15. Remark. Let $L$ be as above. Then the following is not provable in $\mathrm{ZF}$ :

(*) There is a map $p$ which assigns to each definable monotone function $f: L \rightarrow L$ a polynomial $p^{f}(\mathrm{x})$ with coefficients in $L$ such that $p^{f}(a)=f(a)$ for all $a \in L$.

This explains why we have to explicitly mention the parameters $\bar{c}$ in lemma 2.14.

Proof of lemma 2.14. We define the map $p$ by induction on $n$ (the arity of $f$ ). We leave the case $n=0$ to the reader.

Let $f: L^{n+1} \rightarrow L, \bar{c}=\left(c_{1}, \ldots, c_{k}\right) \in L^{k}$. (Wlog assume that $f$ is definable from $\bar{c}$, otherwise set $p^{f ; \bar{c}}=0$.)

Let $A=L \backslash\left\{c_{1}, \ldots, c_{k}, 0,1\right\}$.

We will now apply the induction hypothesis to the functions $f_{\alpha}$ : $L^{n} \rightarrow L$, where $f_{\alpha}(\bar{a})=f(\bar{a}, \alpha)$. For each $\alpha \in L$ we thus get a term $\tau_{\alpha}=p^{f_{\alpha}}$. We will show that only finitely many different terms actually appear, and that they can be combined to yield a term $p^{f}$. The cases $\alpha \in A$ and $\alpha \notin A$ have to be treated in different ways.

To save us some cases distinctions, we agree that $c_{k+1}=0, c_{k+2}=1$.

For each $\alpha \in\left\{c_{1}, \ldots, c_{k+2}\right\}$ the function $f_{\alpha}: L^{n} \rightarrow L, f_{\alpha}(\bar{a})=$ $f(\bar{a}, \alpha)$ is definable from $\bar{c}=\left(c_{1}, \ldots, c_{k}\right)$. Let

$$
\tau_{\alpha}^{f ; \bar{c}}\left(\overline{\mathbf{x}}^{n} ; \overline{\mathbf{s}}^{k} ; \overline{\mathrm{t}}^{3^{n+1}}\right)=p^{f_{\alpha}, \bar{c}}\left(\overline{\mathbf{x}}^{n} ; \overline{\mathbf{s}}^{k} ; \overline{\mathrm{t}}^{3^{n}}\right)
$$

(so the indeterminates $\mathrm{t}_{3^{n}+1}, \ldots, \mathrm{t}_{3^{n+1}}$ do not appear in $\tau_{\alpha}^{f, \bar{c}}$.) By our inductive assumption we know for all $\bar{a} \in L^{n}$ :

$$
\tau_{\alpha}^{f ; \bar{c}}\left(\bar{a}^{n} ; \bar{c}^{k} ; \bar{d}^{3^{n+1}}\right)=f_{\alpha}(\bar{a})=f(\bar{a}, \alpha)
$$

for all $\alpha \in\left\{c_{1}, \ldots, c_{m+2}\right\}$, whenever $\bar{d}$ is independent over $\bar{c}$.

Let

$$
\left.\tau_{\text {constant }}^{f ; \overline{\mathbf{x}}}, \overline{\mathrm{x}}_{n+1} ; \bar{s}^{k} ; \bar{d}^{3^{n+1}}\right)=\bigvee_{i=1}^{m+2}\left[\chi_{c_{i}}\left(\mathrm{x}_{n+1}, \overline{\mathrm{t}}^{3}\right) \wedge \tau_{c_{i}}^{f ; \bar{c}}\left(\overline{\mathbf{x}}^{n} ; \overline{\mathbf{s}}^{k} ; \overline{\mathrm{t}}^{3^{n+1}}\right)\right]
$$


Hence we have for all $\bar{a} \in L^{n}$ :

$\tau_{\text {constant }}\left(\bar{a}^{n}, \alpha ; \bar{c}^{k} ; \bar{d}^{3^{n+1}}\right)= \begin{cases}f(\bar{a}, \alpha) & \text { if } \alpha \in\left\{c_{1}, \ldots, c_{k}, 0,1\right\} \\ f(\bar{a}, 0) & \text { if } \alpha \in A=L \backslash\left\{c_{1}, \ldots, c_{k}, 0,1\right\} .\end{cases}$

whenever $\bar{d}$ is independent over $\bar{c}$. This follows easily from equation $(-1-)$ ) and the monotonicity of $f$.

For each $\alpha \in A$ the function $f_{\alpha}: L^{n} \rightarrow L, f_{\alpha}\left(\bar{a}^{n}\right)=f\left(\bar{a}^{n}, \alpha\right)$ is definable from $(\alpha, \bar{c})$. Hence the polynomial $p^{f_{\alpha} ; \alpha, \bar{c}}\left(\overline{\mathbf{x}}^{n} ; \mathbf{x}_{n+1}, \overline{\mathbf{s}}^{k} ; \bar{t}^{3^{n}}\right)$ satisfies for all $\bar{a} \in L^{n}$

$$
p^{f_{\alpha} ; \alpha, \bar{c}}\left(\bar{a} ; \alpha, \bar{c}^{k} ; \bar{d}\right)=f(\bar{a}, \alpha)
$$

for all $\alpha \in A$, whenever $\bar{d}$ is independent over $\alpha, \bar{c}$.

We claim that the function $\alpha \mapsto p^{f_{\alpha} ; \alpha, \bar{c}}\left(\overline{\mathbf{x}} ; \mathbf{x}_{n+1}, \overline{\mathbf{s}} ; \overline{\mathrm{t}}\right)$ is actually constant on $A$. The reason is that $A$ is disjoint to the set of parameters from which $f$ is defined.

More formally, let $\alpha, \beta \in A$. We will show $p^{f_{\alpha} ; \alpha, \bar{c}}=p^{f_{\beta} ; \beta, \bar{c}}$. Let $\pi: L \rightarrow L$ be an automorphism that fixes $\left\{c_{1}, \ldots, c_{k}, 0,1\right\}$ pointwise, and maps $\alpha$ to $\beta$. Then $\pi f=f$, by fact 2.9.

Hence

$$
\begin{aligned}
p^{f_{\alpha} ; \alpha, \bar{c}} & =p^{\pi\left(f_{\alpha}\right) ; \pi(\alpha), \pi(\bar{c})} & & \text { by induction hypothesis, lemma 2.14(3) } \\
& =p^{(\pi f)_{\pi(\alpha) ; \beta, \bar{c}}} & & \text { since } \pi\left(f_{\alpha}\right)=(\pi f)_{\pi(\alpha)} \\
& =p^{f_{\beta} ; \beta, \bar{c}} & & \text { since } \pi f=f
\end{aligned}
$$

We will write $\tau_{\text {raw }}^{f ; \bar{c}}\left(\overline{\mathrm{x}}, \mathrm{x}_{n+1} ; \overline{\mathrm{s}} ; \overline{\mathrm{t}}\right)$ for the common value of this function. So we can rewrite equation (-3-) as: for all $\bar{a} \in L^{n}, \alpha \in A$ :

$$
\tau_{\text {raw }}^{f ; \bar{c}}(\bar{a}, \alpha ; \bar{c} ; \bar{d})=f(\bar{a}, \alpha)
$$

whenever $\bar{d}$ is independent over $\bar{c}$.

The restriction that $\bar{d}$ has to be independent not only of $\bar{c}$ but also of $\alpha$ is inconvenient. We get rid of it with the following "error correction" device: Let

$$
\begin{aligned}
& \sigma_{1}^{f ; \bar{c}}\left(\overline{\mathbf{x}}^{n}, \mathbf{x}_{n+1} ; \overline{\mathbf{s}} ; \mathrm{t}_{1}, \ldots, \mathrm{t}_{3^{n+1}}\right)=\tau_{\text {raw }}^{f ; \bar{c}}\left(\overline{\mathbf{x}}^{n}, \mathbf{x}_{n+1} ; \overline{\mathbf{s}} ; \overline{\mathrm{t}}_{1}, \ldots, \mathrm{t}_{3^{n}}\right) \\
& \sigma_{2}^{f ; \bar{c}}\left(\overline{\mathbf{x}}^{n}, \mathbf{x}_{n+1} ; \overline{\mathbf{s}} ; \mathrm{t}_{1}, \ldots, \mathrm{t}_{3^{n+1}}\right)=\tau_{\text {raw }}^{f ; \bar{c}}\left(\overline{\mathbf{x}}^{n}, \mathbf{x}_{n+1} ; \overline{\mathbf{s}} ; \mathrm{t}_{3^{n}+1}, \ldots, \mathrm{t}_{2 \cdot 3^{n}}\right) \\
& \sigma_{3}^{f ; \bar{c}}\left(\overline{\mathbf{x}}^{n}, \mathbf{x}_{n+1} ; \overline{\mathbf{s}} ; \mathrm{t}_{1}, \ldots, \mathrm{t}_{3^{n+1}}\right)=\tau_{\text {raw }}^{f ; \bar{c}}\left(\overline{\mathbf{x}}^{n}, \mathbf{x}_{n+1} ; \overline{\mathbf{s}} ; \mathrm{t}_{2 \cdot 3^{n}+1}, \ldots, \mathrm{t}_{3 \cdot 3^{n}}\right)
\end{aligned}
$$

and let

$$
\tau_{\text {clean }}^{f ; \bar{c}}=\mu\left(\sigma_{1}^{f ; \bar{c}}, \sigma_{2}^{f ; \bar{c}}, \sigma_{3}^{f ; \bar{c}}\right),
$$

where $\mu(\mathrm{x}, \mathrm{y}, \mathrm{z})$ is a majority term, i.e., $\mu$ satisfies

$$
\mu(a, a, b)=\mu(a, b, a)=\mu(b, a, a)=a
$$

for all $a, b \in L$, see definition 2.12 .

Let

$$
\tau_{\text {bounded }}^{f ; \overline{\mathrm{c}}}\left(\overline{\mathrm{x}}^{n}, \mathrm{x} ; \overline{\mathbf{s}} ; \overline{\mathrm{t}}\right)=\tau_{\text {clean }}^{f ; \overline{\mathrm{c}}}\left(\overline{\mathrm{x}}^{n}, \mathrm{x} ; \overline{\mathrm{s}} ; \overline{\mathrm{t}}\right) \wedge \tau_{1}^{f ; \bar{c}}\left(\overline{\mathrm{x}}^{n} ; \overline{\mathrm{s}} ; \overline{\mathrm{t}}\right) \wedge \chi_{A}^{\bar{c}}\left(\mathrm{x}, \overline{\mathrm{t}}^{3}\right) .
$$


We claim that $\tau_{\text {bounded }}^{f ; \overline{\mathrm{x}}}\left(\overline{\mathrm{x}}^{n}, \mathrm{x}_{n+1} ; \overline{\mathrm{s}}^{k} ; \overline{\mathrm{t}}^{3^{n+1}}\right)$ satisfies

$$
\tau_{\text {bounded }}^{f ; \bar{a}}\left(\bar{a}^{n}, \alpha ; \bar{c}^{k} ; \bar{d}^{3^{n+1}}\right)= \begin{cases}f\left(\bar{a}^{n}, \alpha\right) & \alpha \in A \\ 0 & \alpha \in\left\{c_{1}, \ldots, c_{k}, 0\right\} \\ \leq f(\bar{a}, 1) & \alpha=1\end{cases}
$$

for any $\bar{a} \in L^{n}, \alpha \in L$, whenever $\bar{d}$ is independent over $\bar{c}$.

This is clear if $\alpha \in\left\{c_{1}, \ldots, c_{k}, 0\right\}$. For $\alpha=1$ recall that $\tau_{1}^{f ; \bar{c}}(\bar{a} ; \bar{c} ; \bar{d})=$ $f(\bar{a}, 1)$.

Finally, let $\alpha \in A$, and assume that $\bar{d}$ is independent over $\bar{c}$. Let

$$
\bar{d}_{1}=\left(d_{1}, \ldots, d_{3^{n}}\right) \quad \bar{d}_{2}=\left(d_{3^{n}+1}, \ldots, d_{2 \cdot 3^{n}}\right) \quad \bar{d}_{3}=\left(d_{2 \cdot 3^{n}+1}, \ldots, d_{3 \cdot 3^{n}}\right)
$$

Now since all the $d_{j}$ are distinct, at least two among $\bar{d}_{1}, \bar{d}_{2}, \bar{d}_{3}$ do not contain $\alpha$ and hence are independent over $\left\{c_{1}, \ldots, c_{k}, \alpha\right\}$. So by equation (-4-), at least two of the equations

$$
f_{\alpha}(\bar{a})=\sigma_{\ell}^{f ; \bar{c}}\left(\bar{a}, \alpha ; \bar{c} ; \bar{d}^{3^{n+1}}\right) \quad \ell=1,2,3
$$

are true, hence we have (by the property $(-5-1)$ )

$$
\tau_{\text {clean }}^{f ; \bar{c}}(\bar{a}, \alpha ; \bar{c} ; \bar{d})=f_{\alpha}(\bar{a})
$$

so $\tau_{\text {bounded }}^{f ; \bar{a}}(\bar{a}, \alpha ; \bar{c} ; \bar{d})=f_{\alpha}(\bar{a}) \wedge \tau_{1}(\bar{a} ; \bar{c} ; \bar{d})=f(\bar{a}, \alpha) \wedge f(\bar{a}, 1)=f(\bar{a}, \alpha)$ for all $\alpha \in A$.

This concludes our discussion of the term $\tau_{\text {bounded }}^{f ; \bar{c}}$.

We can now define $p^{f ; \bar{c}}$ as

$p^{f ; \bar{c}}\left(\overline{\mathbf{x}}^{n}, \mathbf{x}_{n+1} ; \overline{\mathbf{s}}^{k} ; \overline{\mathrm{t}}^{3^{n+1}}\right)=\tau_{\text {constant }}^{f ; \overline{\mathrm{x}}}\left(\overline{\mathbf{x}}^{n}, \mathbf{x}_{n+1} ; \overline{\mathbf{s}} ; \overline{\mathrm{t}}\right) \vee \tau_{\text {bounded }}^{f ; \overline{\mathrm{c}}}\left(\overline{\mathbf{x}}^{n}, \mathbf{x}_{n+1} ; \overline{\mathbf{s}} ; \overline{\mathrm{t}}\right)$

From (-2-) and (-6-) we now get the desired property

$$
p^{f ; \bar{c}}(\bar{a}, \alpha ; \bar{c} ; \bar{d})=f(\bar{a}, \alpha)
$$

whenever $\bar{d}$ is independent over $\bar{c}$.

Theorem 2.2 now immediately follows from lemma 2.14.

\section{REFERENCES}

[1] Martin Goldstern and Saharon Shelah. Order-polynomially complete lattices must be LARGE. Algebra Universalis, 39:197-209, 1998.

[2] Hans Kaiser. Interpolation and order polynomially complete lattices. volume 9 of Contributions to general algebra. Hölder-Pichler-Tempsky, Wien, 1995.

[3] Dietmar Schweigert. Zur Theorie der Verbandspolynome. PhD thesis, Technische Universität Wien, 1972.

Institut für Algebra, Technische Universität Wien, Wiedner HauptStrasse 8-10/118.2, A-1040 Wien, Austria

E-mail address: Martin.Goldstern@tuwien.ac.at

Department of Mathematics, Hebrew University of Jerusalem, GiVAT RAM, 91904 Jerusalem, ISRAEL

E-mail address: shelah@math.huji.ac.il 